\title{
Análisis comparativo de Parques Tecnológicos vinculados a Universidades en función del rol de éstas en los ecosistemas de innovación
}

\section{Comparative analysis of Technology Parks linked to Universities based on their role in innovation ecosystems}

\author{
Eloisa Gonçalves da Silva Torlig ${ }^{1}$ \\ eloisatorlig@gmail.com \\ Leopoldo Gomes Muraro 2 \\ leopoldo.Igm@gmail.com \\ Pedro Carlos Resende Junior ${ }^{3}$ \\ pcrj73@gmail.com \\ Edgar Reyes Júnior ${ }^{4}$ \\ prof.edreyes@gmail.com \\ Bruno Rezzoagli 5 \\ brezzoagli@hotmail.com
}

Fecha de recibo: 1/9/2019, Fecha de aceptación: 1/11/2019

\begin{abstract}
RESUMEN
En el presente artículo se realiza un análisis comparativo de parques tecnológicos vinculados a Universidades, en función de las categorías de actuación de estas últimas en los ecosistemas de Innovación identificadas en la bibliografía especializada (articulación de alianzas; desarrollo y transferencia de tecnologías; interconexión y aplicación de conocimientos). Para ello se procedió, por un lado, a recolectar, categorizar y consolidar artículos científicos disponibles en las bases Web of Science y Scielo, y por otro, a comparar los datos oficiales publicados en los sitios electrónicos de dos parques tecnológicos: uno en Brasil, Parque Científico y Tecnológico (PCTec) conectado a la Universidad de Brasilia; y otro en Argentina, Parque Tecnológico del Litoral Centro (PTLC) vinculado a la Universidad Nacional del Litoral, a los fines de contrastar la presencia de tales categorías. Se observa que ambos parques tecnológicos formalmente (normativamente) alcanzan las tres categorías de actuación de las universidades en los ecosistemas de innovación, pero en la práctica sólo la mitad fueron identificadas.
\end{abstract}

\footnotetext{
${ }^{1}$ Universidad de Brasilia, Brasilia, Brasil ${ }^{2}$ Universidad de Brasilia, Brasilia, Brasil ${ }^{3}$ Universidad de Brasilia, Brasilia, Brasil ${ }^{4}$ Universidad de Brasilia, Brasilia, Brasil

${ }^{5}$ Universidad Nacional del Litoral, Santa Fe, Argentina
} 


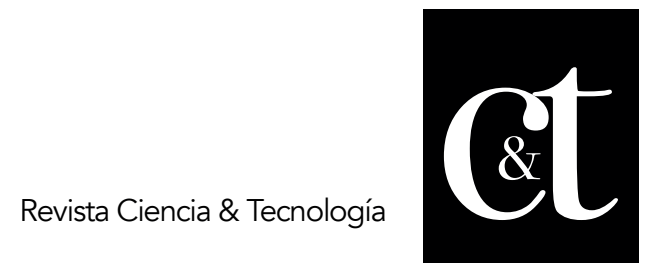

No. 25, 31 de enero de 2020

ISSN impreso: 1390 - 6321

ISSN online: 2661 - 6734

Como sugerencia de estudios futuros, se recomienda investigar el proceso de innovación colaborativa dentro de ecosistemas y estudios empíricos en diferentes parques tecnológicos vinculados a universidades para identificar si las tres categorías identificadas en esa investigación están siendo alcanzadas.

Palabras clave: Parques Tecnológicos, ecosistemas de innovación

\section{ABSTRACT}

In this article a comparative analysis of technological parks linked to Universities is carried out, based on the categories of performance of the latter in the innovation ecosystems identified in the specialized bibliography (alliance articulation; development and transfer of technologies; interconnection and application of knowledge). For this, we proceeded, on the one hand, to collect, categorize and consolidate scientific articles available on the Web of Science and Scielo bases, and on the other, to compare the official data published on the electronic sites of two technology parks: one in Brazil, Science and Technology Park (PCTec) connected to the University of Brasilia; and another in Argentina, Parque Tecnológico del Litoral Centro (PTLC) linked to the Universidad Nacional del Litoral, in order to contrast the presence of such categories. It is observed that both technology parks formally (normatively) reach the three categories of performance of universities in innovation ecosystems, but in practice only half were identified. As a suggestion of future studies, it is recommended to investigate the process of collaborative innovation within ecosystems and empirical studies in different technological parks linked to universities to identify if the three categories identified in that research are being achieved.

Keywords: Technology Parks, innovation ecosystems

\section{Introducción}

En el presente artículo se lleva a cabo una comparación entre dos parques tecnológicos vinculados a universidades (Universidad de Brasilia y Universidad Nacional del Litoral) a los fines de contrastar la actuación de las universidades en los denominados ecosistemas de innovación. Si bien el conocimiento desde siempre ha sido utilizado por la sociedad como base del progreso económico-social; son las sociedades modernas las que han convertido a la ciencia y la tecnología en la base de la economía y de la sociedad (Adán, 2012), otorgándole a la universidad un nuevo rol, además de los roles tradicionales de docencia e investigación, consistente en transferir de manera directa el resultado del conocimiento y la tecnología hacia la industria y hacia la sociedad en general (Herrera-Márquez, Salas-Navarro, Domínguez-Moré, y TorresSaumeth, 2015).

El modelo ecosistémico, en el contexto de pleno auge de nuevas formas de gestión que soportan la interacción de estructuras descentralizadas y asociaciones innovadoras entre universidades, instituciones estatales, organizaciones empresariales o sociales, surge como una propuesta viable para responder a las demandas de una sociedad cada vez más compleja (Arregui y Martin, 2015).

Efectivamente, frente a los problemas complejos que envuelven a la actual sociedad del conocimiento y globalización, las universidades emergen como actores claves llamados a ser fuente permanente de innovación a los fines de dar respuestas a dichas demandas. Tarea que puede llevar a cabo a partir de la articulación con otros actores 


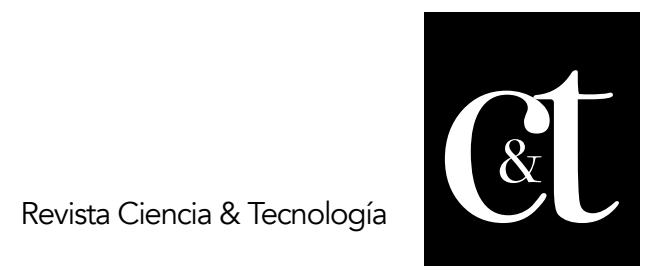

No. 25, 31 de enero de 2020

ISSN impreso: 1390 - 6321

ISSN online: 2661 - 6734

importantes, como la industria, organizaciones de la sociedad civil, actores estatales y otras universidades (Gatica, Soto y Vela, 2005).

Esa comunidad de diversos actores (organizaciones que comparten conocimientos, habilidades y tecnologías) se denomina "ecosistema de innovación", donde cada uno desempeña un papel diferente en un ambiente orientado a las innovaciones y el aprendizaje (Autio \& Thomas, 2014, Iansiti \& Levien, 2004). Los denominados "ecosistemas de innovación" deben comprenderse desde un enfoque en las instituciones (como empresas, universidades, inversores, gobiernos) y a partir de sus interacciones estrechamente entrelazadas (Adner, 2006).

Si bien el concepto de ecosistema ha crecido significativamente en el campo de la gestión de la tecnología y la innovación, el término todavía se utiliza sin una definición clara o un soporte teórico sólido (Adner y Kapoor, 2010; Autio \& Thomas, 2014). Concretamente, se observan diversos conceptos y perspectivas sobre el tema, desde el enfoque de negocios (Iansiti \& Levien, 2004), de servicios (Lusch \& Nambisan, 2015), de emprendedor (Autio \& Thomas, 2014), de redes o de innovación abierta (Carayannis y Campbell, 2012).

No obstante, existe consenso que un ecosistema involucra diversos actores que moldean complejas relaciones a fin de viabilizar desarrollo e innovación tecnológica (Folz, 2014); resultando, consecuentemente, sumamente importante comprender dichas relaciones ya que pueden afectar tanto el equilibrio como la dinámica del ecosistema (Valkokari, 2015). Es por ello que este estudio se apoya, bajo la perspectiva de los ecosistemas de innovación, en dos vertientes: necesidad de avance teórico sobre el tema y comprensión acerca del papel de los actores.

El conocimiento necesario para la implementación de innovaciones está, además de redes de empresas y redes sociales (Folz, 2014), en las universidades e instituciones de investigación; y es justamente por ello que las universidades, en cuanto motores del desarrollo económico regional e innovación tecnológica, pueden desempeñar un papel especial en los ecosistemas de innovación, facilitando la interacción entre la investigación y su aplicación comercial (Youtie \& Shapira, 2008), así como, estimulando la producción y difusión de conocimiento entre regiones (Finegold, 1999).

Indudablemente, la colaboración que la Universidad con las empresas y demás organizaciones constituye un puente que facilita el tránsito hacia un ecosistema de innovación más amplio (Nauwelaers, 2011, Autio \& Thomas, 2014). Es por ello que en este escrito se centra la atención en el papel de la Universidad en los ecosistemas de innovación, en cuanto elemento clave de éstos (Hall, Link \& Scott, 2003).

En este sentido, surgen los parques tecnológicos como un lugar de interacción y formación de alianzas estratégicas entre los actores que participan en los procesos de ciencia y tecnología en busca de innovación, sirviendo de esta manera como un ecosistema de innovación. Neto y Paula (2009) afirman que los parques tecnológicos, como emprendimientos que articulan la interacción entre Universidad, Empresas y Gobierno, pueden representar instrumentos de desarrollo y generación de ingresos, sirviendo como un ecosistema con ciclo completo entre los involucrados, en el cual nuevas formas y los modelos de desarrollo emergen.

En el presente artículo se presentará una revisión de literatura sobre ecosistemas de 3 


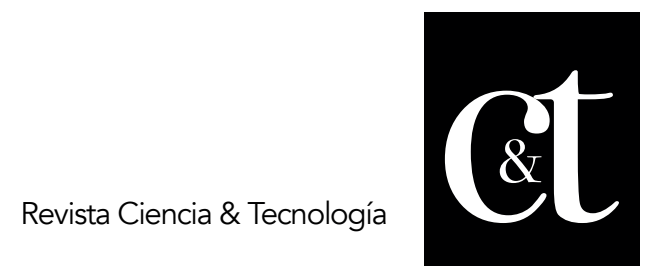

No. 25, 31 de enero de 2020

ISSN impreso: 1390 - 6321

ISSN online: 2661 - 6734

innovación, el método utilizado en la investigación, la discusión sobre el papel de las universidades en los ecosistemas de innovación, una comparación con datos oficiales de un parque tecnológico en Brasil, vinculado a la Universidad de Brasilia (Parque Científico y Tecnológico - PCTec); y otro en Argentina, vinculado a la Universidad Nacional del Litoral (Parque Tecnológico del Litoral Centro - PTLC); y, por último, la agenda de investigación y las consideraciones finales.

\section{Desarrollo}

Ecosistemas de innovación

El concepto ecosistemas de innovación adquirió en estos últimos años una significativa relevancia tanto en el discurso empresarial como gubernamental, aunque fue a mediados de los años 90 cuando surgió dicho constructo (Moore, 1993, 1996), y comenzó a ganar espacio en el campo de investigación de estrategia (Moore, 1996; Iansiti \& Levien, 2004; Teece, 2007). Moore (1993) definió ecosistema de negocios como una comunidad económica que es apoyada por -y que interactúa con- un conjunto de organizaciones e individuos, pertenecientes al mundo de los negocios, que producen bienes y servicios de valor para el cliente. Se observa, entonces, que el ecosistema de negocios está relacionado principalmente con la captura de valor.

Así, basado en el concepto de ecosistemas de negocio, pero a la luz de la creación de valor, se propuso el concepto de ecosistema de innovación, que emergió como un término difuso para describir la creciente complejidad de la innovación, involucrando la interacción entre los actores y sus contextos (Adner \& Kapoor, 2010). Así, los ecosistemas de innovación pueden ser comprendidos como comunidades dinámicas, reunidas intencionalmente, con complejas relaciones, basadas en la colaboración, confianza y cocreación de valor, que comparten tecnologías y complementan competencias, a fin de permitir el desarrollo tecnológico e innovación (Gobble, 2014; Jackson, 2011).

La comprensión actual de los ecosistemas de innovación se caracteriza por un enfoque en las instituciones (como empresas, universidades, inversores, gobiernos) y sus interacciones estrechamente entrelazadas; incluyendo, pues, agentes y relaciones económicas y no económicas como la tecnología, las instituciones, las interacciones sociales y la cultura (Mercan \& Gotkas, 2011, Adner, 2006). En este sentido, un ecosistema de innovación abarca una comunidad de diversos actores, que desempeñan diferentes roles, incluyendo organizaciones que comparten conocimientos, habilidades y tecnologías, en un ambiente orientado a las innovaciones y el aprendizaje (Iansiti \& Levien, 2004).

En una visión sistémica, un ecosistema de innovación consiste en agentes y relaciones económicas, así como agentes y relaciones no económicas, involucrados con otras partes, como tecnologías, instituciones, interacciones sociológicas y culturales, de modo que un ecosistema de innovación es un híbrido de diferentes redes o sistemas (Mercan \& Goktas, 2011). Por su parte, Carayannis et al. (2012) traen una visión abierta de ecosistema de innovación, que combina e integra sistemas y ambientes sociales y naturales, subrayando la importancia de un pluralismo y una diversidad de agentes, actores y organizaciones, dispuestos a producir innovación y conocimiento a lo largo de las redes colaborativas.

Como un proceso sistémico, los ecosistemas de innovación se forman dentro de una red de relaciones interorganizacionales, que promueven la interrelación y la 


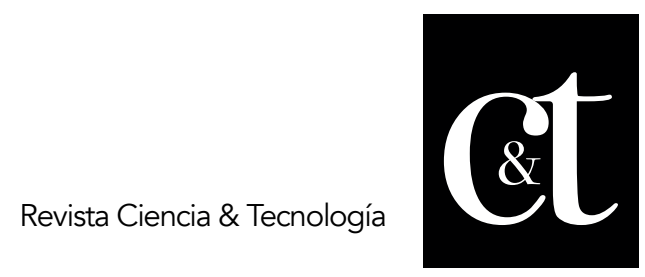

No. 25, 31 de enero de 2020

ISSN impreso: 1390 - 6321

ISSN online: 2661 - 6734

integración del conocimiento de diferentes actores (universidades, centros de investigación, empresas, instituciones y gobiernos), que colaboran y cooperan entre sí, compartiendo conocimiento e intercambiando experiencias, de modo que haya la participación activa y directa de los usuarios en todas las etapas del proceso de innovación (Del Vecchio, Elia, Ndou, Secundo \& Specchia, 2017). Según Carayannis et al. (2012), el ecosistema de innovación parece expandir la idea de red o innovación abierta para abarcar un contexto en red más amplio, incluyendo partes económicas como no económicas, como tecnología, instituciones, interacciones sociológicas y culturales.

Se percibe, así, que el tema viene siendo discutido por diversos autores bajo diferentes perspectivas, como, por ejemplo: Iansiti y Levien (2004) que abordan estrategias organizacionales en relación a los ecosistemas de innovación y ofrecen medidas de desempeño en ecosistemas; Adner (2006) que destaca los factores que restringen el desarrollo de los ecosistemas de innovación; y Autio y Thomas (2014) que abordan las estructuras de gestión de ecosistemas de innovación. Por su parte, Audy y Piqué (2006) abordan diferentes tipos de ecosistemas de innovación, como distritos de innovación, parques científicos y tecnológicos, clusters, ciudades inteligentes, entre otros. Se presenta, así, como foco de este estudio los parques tecnológicos.

\section{Parques Tecnológicos}

El entendimiento de la importancia de la interacción entre distintas organizaciones que - aplicando capacidades científicas, técnicas y sociales en un determinado contexto geográfico- realizan actividades orientadas a la generación, transmisión, difusión, medición y gestión del conocimiento para generar productos y procesos innovadores, ha llevado a la conformación en muchos países de los denominados parques de Ciencia y tecnología (Ondátegui, 2001; Almeida, Santos y Rui, 2008; Ramírez y García, 2010).

Si bien pueden observarse distintas denominaciones para referirse a dicho clusters de organizaciones basadas en el conocimiento, tales como parque de investigación, parque científico, parque tecnológico o parque científico y tecnológico, en este escrito se utiliza la expresión "parque tecnológico" para definir un espacio de innovación, que ofrece condiciones favorables de localización y proporciona soporte técnico, científico e informativo, para aprovechar el proceso de desarrollo de la región y crear el ambiente innovador (Oliveira, 2009). Dicho concepto, conforme Lalkaka y Bishop (1995), tiene su génesis en Estados Unidos con la experiencia pionera del Stanford Research Park, el primer parque científico registrado vinculado a la Universidad de Stanford (López, 2004), que facilitó el surgimiento de la región del Silicon Valley en California.

En ese sentido, Quince, Lobley y Acha (1994) consideran que los Parques Tecnológicos deben promover la creación y el crecimiento de nuevas empresas de base tecnológica; atraer instituciones de investigación gubernamentales y privadas; atraer proyectos de inversión más amplios y realizar la transferencia de tecnología a la industria local.

Para Courson (1997), en visión ecosistémica, los Parques Tecnológicos pueden ser visualizados como una red, pues tal espacio se trata de una organización compleja y evolutiva y dinámica, de modo que haya dos vertientes: i) asociación, en el mismo 


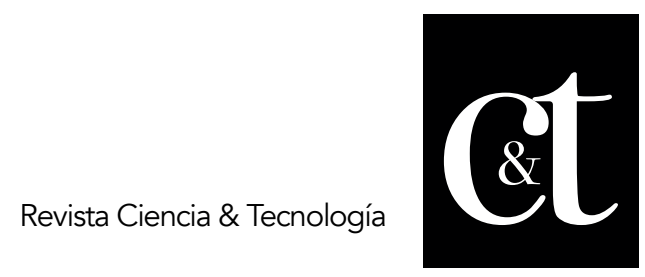

No. 25, 31 de enero de 2020

ISSN impreso: 1390 - 6321

ISSN online: 2661 - 6734

lugar o en el entorno de otros espacios de innovación: universidades, laboratorios de investigación, empresas de alta tecnología y servicios relacionados; ii) vínculos, flujos y relaciones entre estos diversos espacios de innovación.

Cabe aclarar que hay diversas formas de organización de parques tecnológicos, pudiéndose identificar dos ejemplos de modelos relevantes: a) en Europa y en China, hay una tendencia de que el Estado actúe como principal agente, haciendo que los parques sean emprendimientos estatales o mixtos (entidades de derecho privado, pero con participación pública); b) en los Estados Unidos, este papel tiende a ser desempeñado por las universidades, en articulación con el capital privado (Steiner, Cassim y Robazzi, 2012). En Brasil son comunes que los Parques Tecnológicos se ubiquen cerca de las universidades debido a la presencia mayor de generadores de conocimiento y, principalmente, de recursos humanos. Esta proximidad geográfica puede generar sinergias y oportunidades de manera más veloz, comprendiendo una fuente significativa de capital intelectual, ambiente emprendedor e infraestructura compartida (Steiner, Cassim y Robazzi, 2012).

En la doctrina hispano-americana, de forma similar, Castells y Hall (2001: 17) destacan que los Parques Tecnológicos son "centros planificados para la promoción de la industria de alta tecnología, son la realidad de estas transformaciones fundamentales, que redefinen las condiciones y los procesos del desarrollo local y regional", resaltando además que son promovidos por gobiernos centrales, regionales o locales asociados a universidades y empresas privadas que ocupan los espacios resultantes.

De forma complementaria, Rodrigues-Pose (2012) asegura que los parques tecnológicos son una creación, generalmente pública, de una zona geográfica delimitada y destinada a favorecer el desarrollo y aplicación de actividades científicas y tecnológicas, teniendo por objetivo último "la generación de crecimiento económico en el largo plazo en el territorio en el que se instala el PCT "(Rodrigues-Pose 2012, p.7).

\section{Método}

La metodología aplicada se divide en dos partes: (i) una investigación bibliográfica y (ii) un estudio comparado.

En cuanto al procedimiento utilizado en la investigación bibliográfica, se realizó una búsqueda en las bases Scielo y de la Web of Science, considerando el período comprendido entre los años 2008 y $2018^{6}$, utilizando la ecuación "ecosystem*" AND "innovation*" AND "universit*"7, en inglés y portugués -en el título, palabras clave y resumen. Se obtuvieron ciento treinta y siete artículos, los cuales fueron analizados con fines de selección en virtud de los siguientes criterios de selección: a) artículo científico; b) publicado en revistas evaluadas por pares (mínimo B2 o factor de impacto equivalente); c) que reflexione o cuestione la actuación de las universidades en los ecosistemas; e) describir investigaciones teóricas-empíricas. Dicho proceso de selección arrojó un saldo de cuarenta y seis artículos.

En cuanto al interés científico en el tema, es posible identificar que en la base de datos de la Web of Science hubo un aumento considerable de publicaciones a partir de 2015, mientras que, en la base de Scielo, se perciben pocas publicaciones, conforme figura 1: 


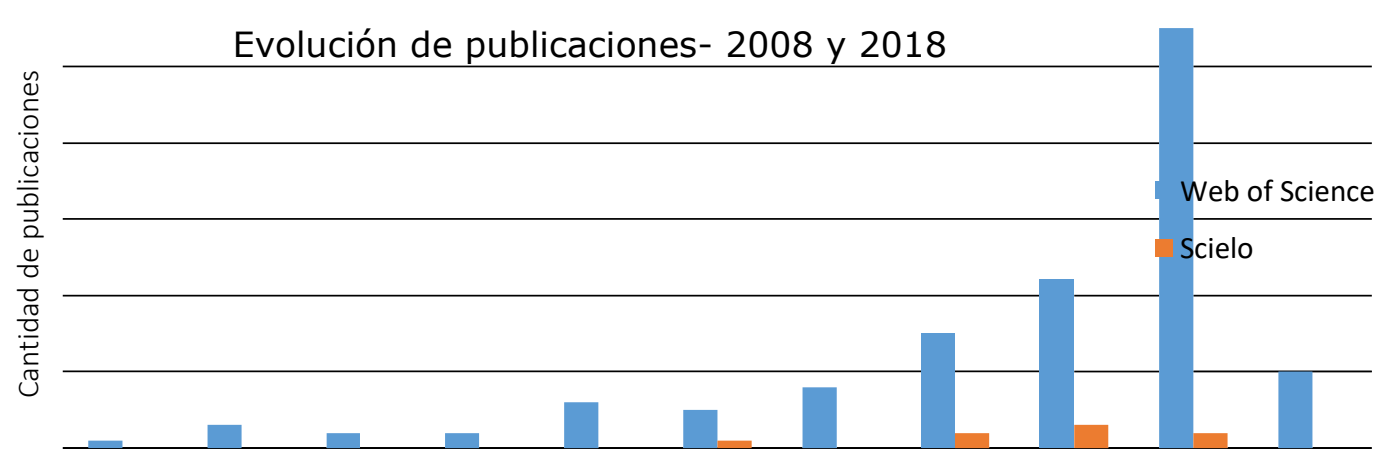

Gráfico 1. Evolución de la cantidad de publicaciones entre 2008 y 2018

Fuente: Elaborado por los autores

En cuanto a los procedimientos de análisis de la investigación bibliográfica, se realizó bibliometría y análisis de contenido, conforme Bardin (2011), para la construcción de las categorías a posteriori. En primer lugar, los artículos fueron divididos por el contexto a ser abordado, luego, se agruparon las principales actuaciones de las universidades evidenciadas en el artículo, que formaron tres categorías temáticas: 1 ) articulación de alianzas; 2) desarrollo y transferencia de tecnología; e 3) interconexión y aplicación de conocimientos. Los resultados fueron analizados por medio del cruce de informaciones de las clasificaciones de los artículos, presentados en la Tabla 1.

En cuanto al procedimiento utilizado en el estudio comparado, se analizaron los datos oficiales que constan en el sitio oficial de internet de dos parques tecnológicos instalados en Países diferentes: (i) el Parque Científico y Tecnológico - PCTec, instalado en la Universidad de Brasilia

-Brasil- y (ii)) el Parque Tecnológico del Litoral Centro, situado en la provincia de Santa Fe, Argentina.

Con base en los datos presentados por los citados parques tecnológicos en los respectivos sitios electrónicos, se agruparon y sintetizaron las informaciones disponibles, posibilitando una contextualización de las actividades desarrolladas dentro de los objetivos oficiales de cada parque.

\footnotetext{
${ }^{6}$ La fecha de corte fue el 31 de marzo de 2018.

7 El símbolo "*" acepta incluir variaciones de las palabras buscadas.
} 


\section{Resultados}

La actuación de las universidades en los ecosistemas de innovación

A través de la revisión bibliográfica, y del cruce de informaciones de las clasificaciones de los artículos, fue posible observar tres categorías temáticas de actuación de las universidades en los ecosistemas de innovación: 1) articulación de alianzas; 2) desarrollo y transferencia de tecnologías; 3) interconexión y aplicación de conocimientos, según figura 2 . Dichas categorías están interconectadas.

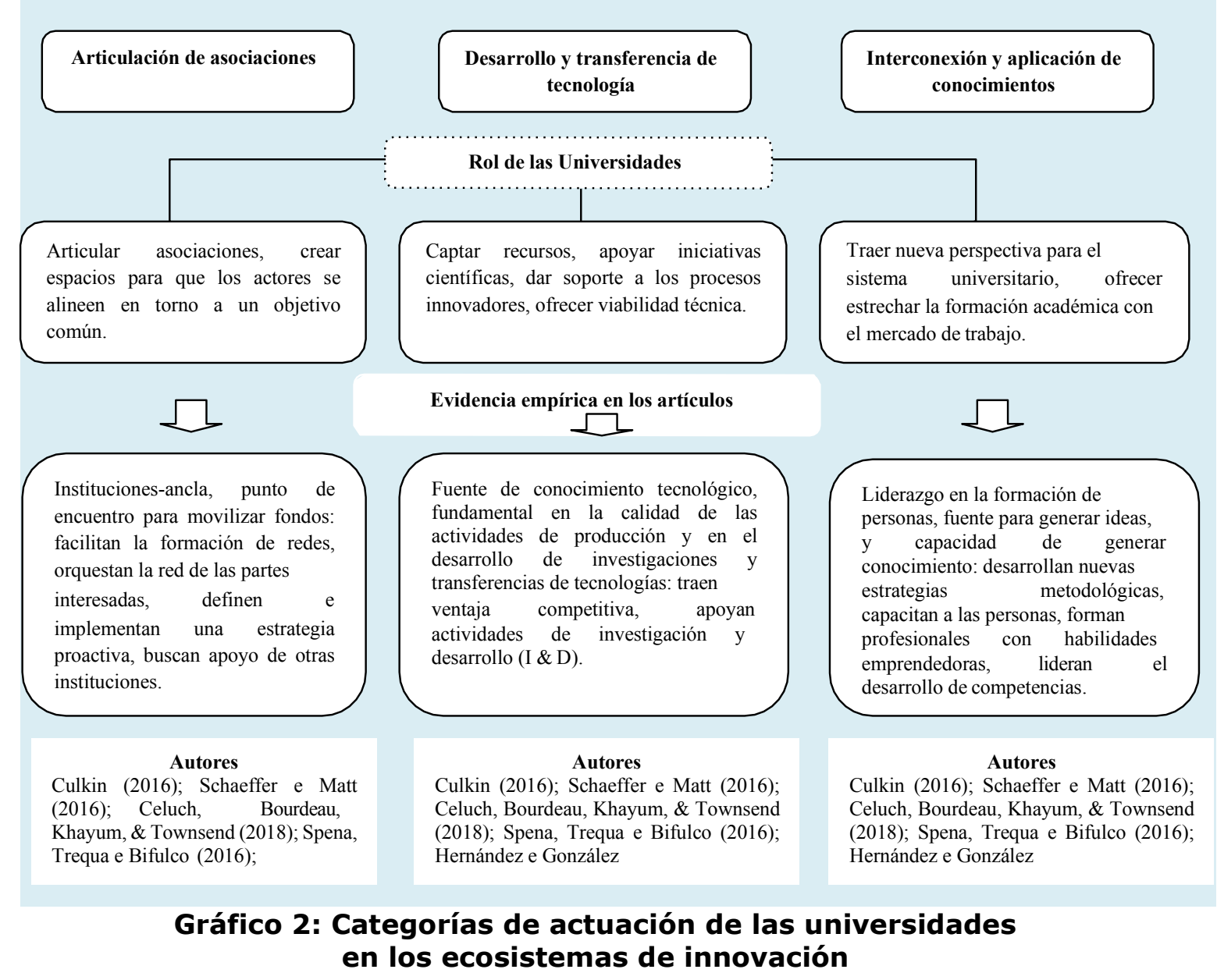

Fuente: Elaborado por los autores

Como se puede apreciar, sobresalen los escritos de ecosistemas de emprendedurismo, asumiendo la Universidad un rol protagónico en el proceso de desarrollo económico y social. Etzkowitz (2003) destaca el contexto de aplicabilidad en el que se materializa esta nueva forma de producción de conocimiento en las universidades orientada a la solución de problemas prácticos, debido a la formación de redes que amplían la interactividad entre universidad y medio industrial. 


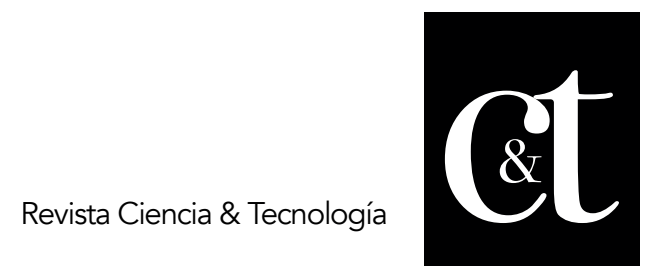

No. 25, 31 de enero de 2020

ISSN impreso: 1390 - 6321

ISSN online: 2661 - 6734

En este sentido, las universidades e institutos de investigación pueden desempeñar un papel crucial en los ecosistemas de innovación, facilitando la interacción entre la investigación y su aplicación comercial, estimulando la producción y difusión de conocimiento entre regiones (Youtie y Shapira, 2008, Finegold, 1999). Como centros de conocimiento, las universidades no sólo producen y acumulan el conocimiento, también existe un intercambio activo con los diversos actores, capaz de generar aprendizaje e innovación, a través de redes e interconexiones de actividades que atraviesan fronteras (Youtie \& Shapira, 2008).

Así, en una perspectiva moderna, la universidad debe ser capaz de construir espacios para alianzas en investigación y educación, que se relacione con la economía del conocimiento, de modo que pueda proporcionar no sólo estructuras de producción de nuevas ideas, tecnologías y dispositivos, y además proporcionar una formación creativa a los futuros profesionales (Kabnob, 2017). Según el autor, las universidades, al formar modelos de redes que gestionen ambientes de aprendizaje creativos, son capaces de hacer su ecosistema emprendedor un lugar de formación y desarrollo de mecanismos eficaces de transferencia de tecnología e innovaciones científicas, representando la base de la competitividad de los países y sus alianzas geopolíticas.

\section{Parque Científico y Tecnológico -PCTec - Brasilia / Brasil}

El Parque Científico y Tecnológico - PCTec fue creado formalmente en 2007, pero lanzado oficialmente el 11 de junio de 2013, encontrándose instalado en la ciudad de Brasilia, Brasil. Este parque fue instalado en la Universidad de Brasilia, buscando actuar como un ecosistema de innovación y promover la interacción de la Universidad con el Gobierno, las empresas, inversores, investigadores, inventores y la comunidad en general.

Es importante destacar que la Universidad de Brasilia es una Fundación Pública Federal, que posee naturaleza autárquica e integra la estructura del Gobierno Federal, o sea, se trata de una universidad pública del Gobierno Federal (Unb, 2018).

De acuerdo con el portal de la Institución, el PCTec (2018) en marzo de 2018 pasó a ser un Órgano Complementario de la Universidad de Brasilia - UnB, que cuenta con autonomía financiera para desarrollar sus actividades. En el pasado, el parque estaba vinculado al Centro de Apoyo al Desarrollo Tecnológico (CDT/UnB) y, dada esta reciente alteración, artículos científicos que abordaban el estudio del Parque Tecnológico de la UnB enfocan como unidad del CDT, siendo este hecho un limitador de la investigación.

En lo que se refiere a los objetivos del parque, de acuerdo con la Resolución 14/2007 del Consejo Directivo de la Fundación Universidad de Brasilia - FUB, se presentan las siguientes finalidades para el PCTec (2018):

a) Atraer inversiones públicas y privadas para la innovación tecnológica, con miras a generar bienestar social y riqueza, así como acercar a la comunidad académica de la UnB de las organizaciones de base tecnológica e innovadoras de alta calificación, creando oportunidades para nuevos proyectos de investigación de punta;

b) Ser un ambiente para la interacción entre empresas, sociedad, gobierno y 


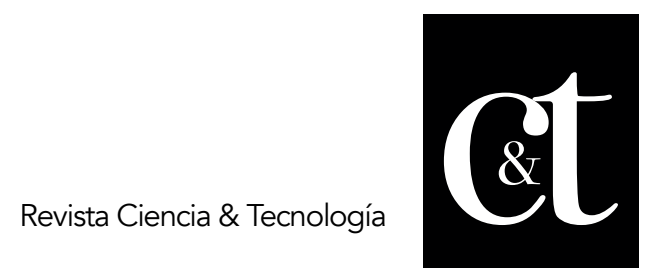

No. 25, 31 de enero de 2020

ISSN impreso: 1390 - 6321

ISSN online: 2661 - 6734

comunidad científica, estableciendo alianzas con instituciones públicas y privadas, nacionales e internacionales con foco en ID\&I;

c) Contribuir a la transformación del Distrito Federal en uno de los grandes centros económicos del país y por el generador de alta tecnología y también de tecnologías sociales;

d) Transformar los resultados de ID\&I en productos, procesos y servicios tecnológicos para atender las demandas de la sociedad y del mercado;

e) Transferir conocimiento científico y tecnológico para empresas de base tecnológica o emprendimientos sociales, por medio del estímulo a la generación y transferencia de conocimiento y tecnologías de la UnB para emprendimientos integrantes del PCTec / UnB, buscando el desarrollo y producción de bienes, procesos y servicios innovadora;

f) Generar nuevos patrones de empleabilidad en las empresas asociadas del PCTec / UnB, especialmente para profesionales de alta calificación;

g) Abrirá nuevas empresas de base tecnológica y base social;

h) Apoyar asociaciones entre la UnB y organizaciones públicas y privadas involucradas con la investigación, el desarrollo social y la innovación tecnológica;

i) Proporcionar oportunidades de prácticas a los alumnos de la UnB, así como facilitar su inserción en el mercado de trabajo;

j) Apoyar el desarrollo de negocios y gestión de los emprendimientos integrantes del PCTec / UnB;

k) Identificar las demandas científicas, tecnológicas y sociales de la región del Distrito Federal que oportunicen la interacción con las unidades académicas y la creación de emprendimientos en el PCTec / UnB.

Se constata, por lo tanto, que el PCTec presenta en su constitución de funcionamiento los elementos que permiten enmarcarlo como un ecosistema de innovación, ya que sirve de espacio y plataforma para promover la interacción entre la universidad, el gobierno y la sociedad ( $y$, (en el caso de las empresas, asociaciones, investigadores, inventores, etc.) para generar y transferir conocimiento tecnológico a partir de investigaciones científicas en la obtención de productos, procesos y servicios innovadores (Moore, 1993, Carayannis y Campbell, 2012, Cusumano \& Gawer, 2002, Iansiti \& Levien, (2004), Teece, 2007).

De acuerdo con las informaciones oficiales que cuentan en el sitio electrónico del PCTec, se agruparon las informaciones relacionadas con sus actividades, resultando en la elaboración de la Tabla 1 abajo: 
Tabla 1. PCTec

\begin{tabular}{|l|l|}
\hline Tipo & Nombre de la Empresa \\
\hline & 1) Brasal \\
Empresas & 2) EcoFossa \\
Residentes & 3) Global IP \\
& 4) Logpress \\
& 5) LoopKey \\
& 6) Maxtera \\
& 7) Quali-A \\
& 8) Redecom \\
\hline Laboratorios & 1) En el sitio electrónico consta que UnB \\
& posee 327 laboratorios en SUS \\
& unidades. \\
\hline
\end{tabular}

Elaborado por los autores

El sitio electrónico del PCTec (2018) presenta en su página datos agrupados en las categorías: el parque, residentes, portal tecnológico, documentos, oportunidades, tesis y monografías de la UnB y hable con nosotros. Dentro de cada categoría, hay datos básicos sobre el tema, uno está en fase de construcción y otro remite al usuario a la página de la UnB.

En la página oficial de la institución (PCtec, 2018) consta poca información acerca de las actividades desarrolladas y no hay división de las empresas o un local para verificar las características y fases de las empresas: no hay mención a pre-incubación, incubación, empresa junior, empresa-anjo, start-up u otro tipo. Tampoco hay un espacio para promover interacción o para que eventuales interesados entren en contacto con integrantes del parque en busca de informaciones y alianzas; solamente la indicación de teléfonos en una pestaña denominada "hable com nosotros".

En este cuadro, las únicas informaciones que cuentan en el sitio oficial del PCTec que demuestran sus actividades son aquellas agrupadas y presentadas en la Tabla 2 (arriba). No hay como afirmar que el parque no posee más socios (alianzas) o si se trata de falta de inclusión de estos socios (alianzas) en el sitio oficial.

\section{Parque Tecnológico Litoral Centro - PTLC - Santa Fe/ Argentina}

El Parque Tecnológico Litoral Centro - PTLC fue creado en 2002 y se encuentra instalado en la ciudad de Santa Fe, Provincia de Santa Fe, Argentina. El PTCL es una sociedad anónima compuesta por acciones de 3 grupos principales: universidad, gobierno y empresas, dentro del modelo de la triple hélice de la innovación (Etzkowitz, 2003).

EI PTLC (2018) es una Sociedad Anónima con Participación Estatal Majoritaria (SAPEM) compuesta por el capital accionario de 12.000 acciones, divididas en 3 Clases:

\section{Clase A}

- científico/tecnológico (acciones de la Universidad Nacional del Litoral); Clase B gubernamental (Provincia de Santa Fe y 2 Municipios: Santa Fe y Paraná); y Clase C empresarial (Confederaciones Generales de Economía e Industria). 
Clase A

Universidad Nacional del Litoral

Clase B

Gobierno de la Provincia Santa

Fe Municipalidad de Santa Fe

Municipalidad de Paraná

Clase C

Confederación

Económica

General de la Industria

General

Confederación

Gráfico 5 - La Sociedad del PTCL

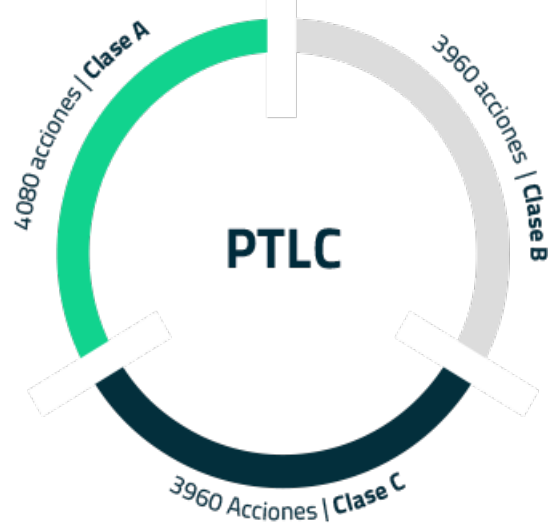

Fuente: PTCL (2018)

En el marco de la hélice de la universidad, en el modelo de la triple hélice de la innovación (Etzkowitz, 2003), el PTLC es integralmente representado por la Universidad Nacional del Litoral, siendo, por lo tanto, considerado para este estudio como el parque tecnológico ligado a la actuación de las universidades en la formación, ejecución y consolidación de ecosistemas de innovación (Kirby, 2006).

En lo que respecta a los objetivos del parque (Estatuto, 2005), se destacan los siguientes:

- Aplicar los objetivos de la promoción y fomento de la innovación tecnológica, establecidos en la Ley Nacional $\mathrm{N} \circ 23877$, las que en el futuro pudieran sustituirla al complementarla.

- Propiciar la integración y complementarización de las actividades industriales de alta tecnología y aspectos productivos, técnicos, comerciales y financieros.

- Incubar y radicar emprendimientos empresariales de base tecnológica.

- Crear a través de la concentración concentrada de establecimientos industriales de base tecnológica, las condiciones que permitan la reducción de los costos de inversión en infraestructura y servicios.

- Reglamentar y administrar Áreas Industriales e Incubadoras de Empresas de Base Tecnológica y promover la instalación en ellas de empresas de bienes y servicios de base tecnológica em armonía con el ambiente y los núcleos urbanos.

- Alentar los procesos de capacitación de recursos humanos, empresarios y laborales y el crecimiento del empleo industrial de alta calificación técnica por medio de acciones comunes.

- Participar en las actividades de asociaciones científicas y tecnológicas, nacionales y / o internacionales.

De acuerdo con las informaciones oficiales disponibles en el sitio electrónico, el PTCL presenta los siguientes datos relacionados con sus actividades. 


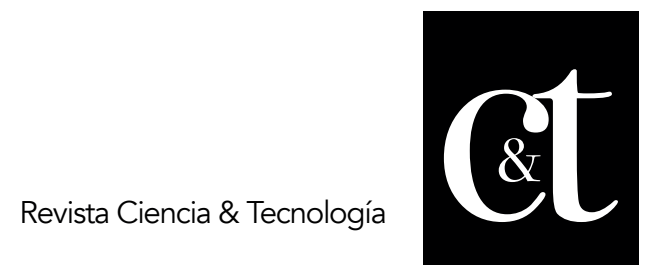

No. 25, 31 de enero de 2020

ISSN impreso: 1390 - 6321

ISSN online: 2661 - 6734

Tabla 2. Informaciones oficiales sitio electrónico PTCL

\begin{tabular}{|l|l|}
\hline Tipo & \multicolumn{1}{|c|}{ Nombre de la Empresa } \\
\hline \multirow{3}{*}{ TICs } & 1) Asso \\
& 2) Server EBT \\
& 3) SIGES \\
\hline PREINCUBADAS & 4) PUNTOSIM - Sistemas Integrados y Móviles \\
\hline \multirow{3}{*}{ 1) Redmaco } \\
2) Plexar Technologies \\
\hline 1) Alytix S.A. \\
& 2) ACRONEX \\
& 3) Rock River Laboratory \\
& 4) Lipomize Nanobio Solutions \\
& 5) NOVARTEK \\
& 6) PPST Argentina \\
\hline RADICADAS & 1) Horian Carbonfe \\
& 2) Lipomize Nanobio Solutions \\
& 3) CLORAR Ingeniería S.A. \\
\hline 1) ZOOVET \\
2) ZELLTEK S.A
\end{tabular}

Fuente: Elaborado por los autores.

El sitio electrónico del PTCL (2018) presenta variados datos organizados inicialmente en las categorías: Quiénes somos, Cómo instalarse, Empresas, Noticias, Ubicación y Contacto. Adentrando en cada una de las páginas constan datos sobre los respectivos tópicos, siendo que hay información en todas las páginas. Se resalta, por fin, que hay un formulario electrónico para ser llenado por posibles interesados en contactar el parque tecnológico, siendo una forma simple de acceso e interacción.

\section{Conclusiones}

El estudio comparado de los parques tecnológicos vinculados a las Universidades Nacional del Litoral (Argentina) y de Brasilia (Brasil), con base en los datos oficiales presentados en la página de cada institución, permite constatar que el Parque Tecnológico del Litoral Centro (PTLC) en comparación con el Parque Científico Tecnológico (PCTec) presenta más datos sobre sus actividades (describe sus acciones, posee espacio interactivo), dándoles a los ciudadanos, investigadores y empresas interesadas mayores posibilidades de conocer el parque y su ecosistema de innovación.

Desde el punto de vista formal, ambos parques presentan sus estructuras normativas dentro del constructo de los ecosistemas de innovación. Los objetivos descritos en los documentos oficiales que constituyeron tanto el PTLC como el PCTec describen acciones típicas de los ambientes innovadores, buscando promover articulaciones e interacciones entre actores, desarrollo de investigaciones y transferencia de tecnología.

Los datos oficiales del PCTec posibilitan señalar que hay interacción en el parque de 8 empresas, sin especificar cuáles son las actividades realizadas, el tipo de situación 


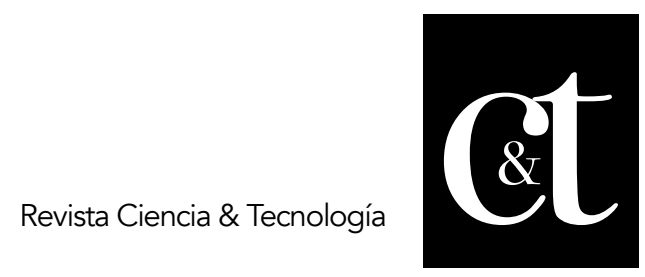

No. 25, 31 de enero de 2020

ISSN impreso: 1390 - 6321

ISSN online: 2661 - 6734

de las empresas y si se han logrado resultados sobre productos, procesos o servicios innovadores. En la página del PTLC, los datos apuntan que hay interacción de 17 empresas, divididas en categorías y se demuestran varias actividades realizadas, sin embargo, no hay tampoco presentación de los resultados innovadores.

Como estudio comparado, se verifica que formalmente ambos parques tecnológicos presentan en sus objetivos sociales las tres categorías de actuación de las universidades en los ecosistemas de innovación: i) articulación de alianzas; ii) desarrollo y transferencia de tecnologías; iii) interconexión y aplicación de conocimientos. Los documentos que constituyen los parques presentan de forma exhaustiva las acciones que deben ser desarrolladas dentro de cada categoría.

Sin embargo, ante el análisis de los datos oficiales presentados en los respectivos casos, se constata que la primera categoría es alcanzada, la segunda no y la tercera parcialmente. De conformidad con las informaciones consolidadas, hay demostración de que los parques están promoviendo articulación de alianzas e interconexión de conocimientos, una vez que cada uno indica empresas actuando en su espacio; pero no se han identificado datos que permitan medir si hay desarrollo y transferencia de tecnología y aplicación de conocimientos.

Finalmente, se recomienda para futuros estudios investigar el proceso colaborativo de innovación dentro de los ecosistemas, así como estudios empíricos en otros parques tecnológicos vinculados a universidades a los fines de constatar la presencia de las tres categorías identificadas, con base en la investigación bibliográfica, en este trabajo.

\section{Referencias bibliográficas}

Adner, R. (2006). Match your innovation strategy to your innovation ecosystem. $H$ Harvard business review, 84(4), 98.

Adner, R., \& Kapoor, R. (2010). Value creation in innovation ecosystems: How the structure of technological interdependence affects firm performance in new technology generations. Strategic management journal, 31(3), 306-333.

Audy J. \& adnerPiqué J. (2006). Dos Parques Científicos e Tecnológicos aos Ecossistemas de Inovação.

Autio, E. \& Thomas, L. (2014). Innovation ecosystems. The Oxford handbook of innovation management, 204-288.

Bardin, L. (2011). Análise de conteúdo. 3. reimp. Lisboa: Ediçoes, 70.

Carayannis, E. G., Barth, T. D., \& Campbell, D. F. (2012). The Quintuple Helix innovation model: global warming as a challenge and driver for innovation. Journal of Innovation and Entrepreneurship, 1(1), 2.

Castells, M. y Hall, P. (2001). Tecnópolis del mundo. La formación de los complejos industriales del siglo XXI. Alianza: Madrid. 


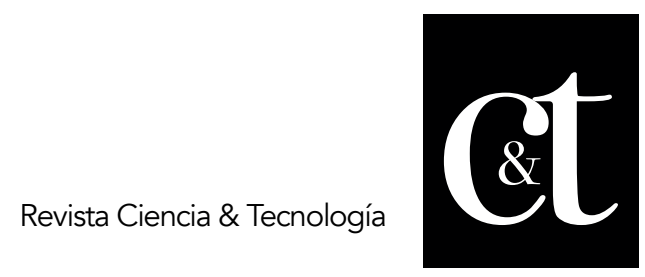

No. 25, 31 de enero de 2020

ISSN impreso: 1390 - 6321

ISSN online: 2661 - 6734

Celuch, K., Bourdeau, B., Khayum, M., \& Townsend, L. (2017). The role of the university in accelerated learning and innovation as a regional ecosystem integrator. Journal of Research in Innovative Teaching \& Learning, 10(1), 3447.

Courson, J. D. (1997). Espaço urbano e parques tecnológicos europeus. Parques tecnológicos e meio urbano: artigos e debates. Brasília, DF: ANPROTEC, 7784.

Cusumano, M. A., \& Gawer, A. (2002). The elements of platform leadership. MIT Sloan management review, 43(3), 51.

Del Vecchio, P., Elia, G., Ndou, V., Secundo, G., \& Specchia, F. (2017). Living Lab as an Approach to Activate Dynamic Innovation Ecosystems and Networks: An Empirical Study. International Journal of Innovation and Technology Management, 14(05), 1750024.

Etzkowitz, H. (2003). Innovation in innovation: The triple helix of university-industrygovernment relations. Social science information, 42(3), 293-337.

Finegold, D. (1999). Creating self-sustaining, high-skill ecosystems. Oxford review of economic policy, 15(1), 60-81.

Folz, C. (Ed.). (2014). Ecossistema Inovação. Brasília: Embrapa.

Gobble, M. M. (2014). Charting the innovation ecosystem. ResearchTechnology Management, 57(4), 55-59.

Hall, B. H., Link, A. N., \& Scott, J. T. (2003). Universities as research partners. Review of Economics and Statistics, 85(2), 485-491.

Iansiti, M., \& Levien, R. (2004). Strategy as ecology. Harvard business review, 82(3), 68-81. Jackson, D. J. (2011). What is an innovation ecosystem. National Science Foundation, 1.

Kirby, D. A. (2006). Creating entrepreneurial universities in the UK: Applying entrepreneurship theory to practice. The Journalof Technology Transfer, 31(5), 599-603.

Lalkaka, R., \& Bishop, J. (1995, September). Technology parks and business incubators: the potential of synergy. In 32th World Conference on Science Parks, Beijing.

Lusch, R. F., \& Nambisan, S. (2015). Service innovation: A service-dominant logic perspective. MIS quarterly, 39(1).

Mercan, B., \& Goktas, D. (2011). Components of innovation ecosystems: a crosscountry study. International Research Journal of Finance and Economics, 76(16), 102-112. 


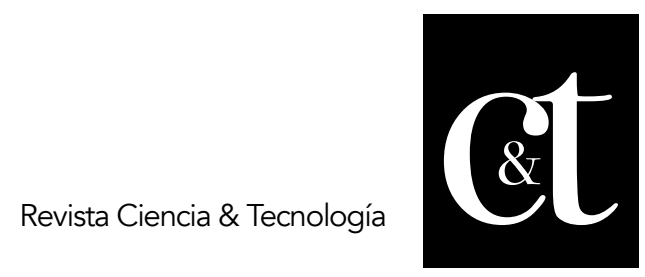

No. 25, 31 de enero de 2020

ISSN impreso: 1390 - 6321

ISSN online: 2661 - 6734

Moore, J. F. (1993). Predators and prey: a new ecology of competition. Harvard business review, 71(3), 75-86.

Moore, J. F. (1996). The death of competition: leadership and strategy in the age of business ecosystems (p. 297). New York: Harper Business.

Nauwelaers, C. (2011). Intermediaries in regional innovation systems: role and challenges for policy. In P. Cooke (Ed.), Handbook of regional innovation and growth (pp. 467-481). Cheltenham: Edward Elgar.

Neto, R. J. \& PAULA, E. (2009). Indicadores de avaliação de desempenho para o parque científico e tecnológico da PUCRS-TECNOPUC, na percepção de seus principais stakeholders. Seminário nacional de parques tecnológicos e incubadoras de empresas, 19.

Oliveira, P. S. (2009) A Política de Desenvolvimento da Biotecnologia no Brasil e o Papel estratégico dos Parques Tecnológicos para o progresso da Bioindústria. Dissertação de Mestrado apresentada à PUC-SP, São Paulo.

Quince, R., Lobley, D., \& Acha, V. (1997). Parques tecnológicos e redes de cooperação. Guedes, F.; Formica, P. A economia dos parques tecnológicos. Rio de Janeiro: Anprotec, 97-110.

Rodrigues-Pose, A. (2012). Los parques tecnológicos en América Latina. Un análisis de la situación actual. BID.

Spena, T. R., Trequa, M., \&Bifulco, F. (2016). Knowledge Practices for an Emerging Innovation Ecosystem. International Journal of Innovation and Technology Management, 13(05), 1640013.

Steiner, J. E., Cassim, M. B., \& Robazzi, A. C. (2008). Parques tecnológicos: ambientes de inovação. Revista IEA. USP. São Paulo.

Teece, D. J. (2007). Explicating dynamic capabilities: the nature and microfoundations of (sustainable) enterprise performance. Strategic management journal, 28(13), 1319-1350.

Valkokari, K. (2015). Business, innovation, and knowledge ecosystems: How they differ and how to survive and thrive within them. Technology Innovation Management Review, 5(8).

Youtie, J., \& Shapira, P. (2008).Building an innovation hub: A case study of the transformation of university roles in regional technological and economic development. Research policy, 37(8), 1188-1204. 